\title{
Device and Service Descriptions in Personal Distributed Environments
}

\author{
Suparna De, Klaus Moessner \\ Centre for Communication Systems Research \\ University of Surrey, Guildford, Surrey, GU2 7XH, United Kingdom \\ \{S.De, K.Moessner\}@surrey.ac.uk
}

\begin{abstract}
The current research on context-aware systems in ubiquitous environments opens a number of interlinked research challenges. On the lowest level of such systems, discovery mechanisms and flexible semantic descriptions of available devices and services form the basis for end-user service personalization. The challenge is to design a description model that leverages implicit semantic information obtained, while being cognizant of resource constraints of a device. To encounter the different device characteristics and personalization challenges, this paper proposes a device and service description approach that provides a high level contextual view of device information. The work has been performed as part of the Personal Distributed Environment concept, also described in the paper. Further, a user-centric view of multiple user interface devices to access services in a heterogeneous and dynamic networked environment has been implemented by extending UPnP device discovery. A comparison with existing state of the art approaches concludes the work.
\end{abstract}

Keywords- device and service description, multimodal user interfaces, ubiquitous environments.

\section{Introduction}

Current mobile communication environments are characterized by the dynamic availability of devices and services. The rise in the use of wirelessly connected mobile devices such as laptops, cell-phones and PDAs has led to the concept of the Personal Distributed Environment (PDE) [1]. A user's view of the PDE encompasses any device that he can have control over, as opposed to and in addition to explicit ownership. The vision of provisioning services that are relevant to the end-user in a given context entails that such communication environments must be able to collect a wide range of context information and use it to achieve the stated goal.

One of the main subsets of context information that needs to be considered in dynamic, heterogeneous environments is device and service description. The PDE encompasses many service categories, viz. intelligent services (e.g. wake-up call service), Internet or m-commerce services and multimodal services. This paper concentrates on the subset of multimodal services. This set facilitates user interaction and it considers the behavior of the service which is determined by the capabilities and characteristics of the device. For instance, text output service available on a printer or on a screen.

Descriptions must provide enough information to differentiate between similar service offerings. For instance, voice recording software on a device supports 'audio input' modality and the playback facility provides 'audio output'. The basic software information can be qualified with supported formats, viz. allowed file types such as mp3, wma etc. The speaker and microphone characteristics add another layer of refinement and constraint to these services. For the realization of a meaningful dynamic view of available modalities within the user environment, the design and implementation of the descriptions should provide templates for including such information for a wide range of devices.

Current research consists of two distinct approaches for device and service descriptions. The first of these aims to extend standards such as CC/PP [2] and UAProf [3] primarily through the introduction of new attribute vocabularies. However, these efforts suffer from the restricted hierarchy and lack of range specification of CC/PP. The other approach seeks to define new description languages to improve the device discovery and service delivery process. The description model proposed in this paper aims to bridge the gap between device discovery and description steps within 
the PDE by leveraging the implicit information obtained during the discovery process and extending it, while maintaining independence from service domains and being cognizant of resource constraints on mobile devices.

The rest of the paper is organized as follows: current state of the art on device descriptions is analyzed in section 2. Section 3 provides an overview of identified challenges. Section 4 presents an overview of the PDE framework. The description concept is presented in section 5, which is followed by implementation aspects in section 6. The work proposed in this paper is compared to other approaches in section 7 . Conclusions are drawn in section 8 .

\section{Related Work}

There have been several efforts in the research community to describe the capabilities of devices, either as stand alone description efforts (with varied aims, e.g. content adaptation) or as part of a proprietary discovery and description solution. Prominent amongst the $\mathrm{W} 3 \mathrm{C}$ initiatives are $\mathrm{CC} / \mathrm{PP}$ and UAProf specifications.

The CC/PP description template consists of three major categories: terminal hardware, software and browser. The primary objective of this categorization is to enable web servers to tailor content presentation for the client web browser. Application of this template is not intuitive for building comprehensive descriptions. At a first instance, it cannot address device composition as it has a single hierarchy level (root and its children). It also cannot be used for providing format information as it doesn't define a range of values that an attribute may be assigned [4].

UAProf describes a sample vocabulary for describing WAP device profiles. It is tailored to the needs of WAP devices and doesn't scale well to more complex devices such as notebook computers. There are also no means of expressing network connection characteristics. However, as identified in [5], it offers an interesting mechanism of devices publishing their capabilities to a web repository from where it can be retrieved by web servers to tailor web content.

The description model proposed in [4] models a device as hardware or part of hardware. The hierarchical XML schema also allows for specifying a range of attributes. Device types are based on the defined basic types, such as storage, speed, connector, from which 'specTypes' can be defined to indicate specific device characteristics. The model is a primarily hardware centric approach and caters to terminals and its hardware sub-components (such as memory, motherboard etc.) though specific extensions have been defined for describing printers. Though comprehensive in this sense, the template would not handle device types that cannot be built up from the defined basic types, for instance, communication devices such as routers.

Another research effort [6] extends the CC/PP standard by introducing new components in the CC/PP profile. These include quality of service, type of service, function, location, accessible time and additional information, with each part being described by a profile. The function profile is based on the DAML-S approach and details the service inputs, outputs, preconditions and effects. The location profile includes both virtual and geographical location information. However, this approach doesn't have provision for specifying a range of attributes for service function or default values for the same.

\section{Challenges}

Existing approaches cannot be directly applied to the PDE. Based on the review of current state of the art, this section identifies challenges that need to be addressed by a device and service description framework. These challenges arise from the heterogeneity of possible devices, alignment to the discovery process and needed support for automated reasoning.

Generic template

The heterogeneity of devices in the PDE necessitates the development of a generic model that can describe a wider set of devices providing user interaction. Such a template should be sufficiently generic to capture the features of devices capable of providing the means for user interaction, rather than describing the mere hardware capabilities and categorization of devices.

\section{Device-service links}

Service descriptions can provide annotated information on supported formats for offered services, which can be further refined by hardware characteristics. In addition, the service information should provide information on the static properties (e.g. type, provider) as well as the service interface (methods that can be called and parameters) to provide a complete view of the description [7].

\section{Leveraging discovery mechanisms}

Industry standards for resource discovery in ad-hoc environments incorporate some level of descriptions for advertising service offerings. There is a need to build a higher level description model that leverages this implicit information obtained during the discovery 
process, without necessitating already resource constrained devices to host descriptions in new languages or formats.

Ontology support

Descriptions should provide a foundation for automated mapping to ontologies. This would aid in reasoning through an inference engine to provision services relevant to the end user.

\section{PDE framework overview}

The device descriptions presented in this paper form part of the research activity aimed at developing a Personal Assistant Agent (PAA) within the PDE. This section presents a brief overview of the PAA and PDE concepts, focusing on how the description activity fits within the PAA. Figure 1 shows a high-level view of the framework. A more comprehensive description of the framework can be found in [1].

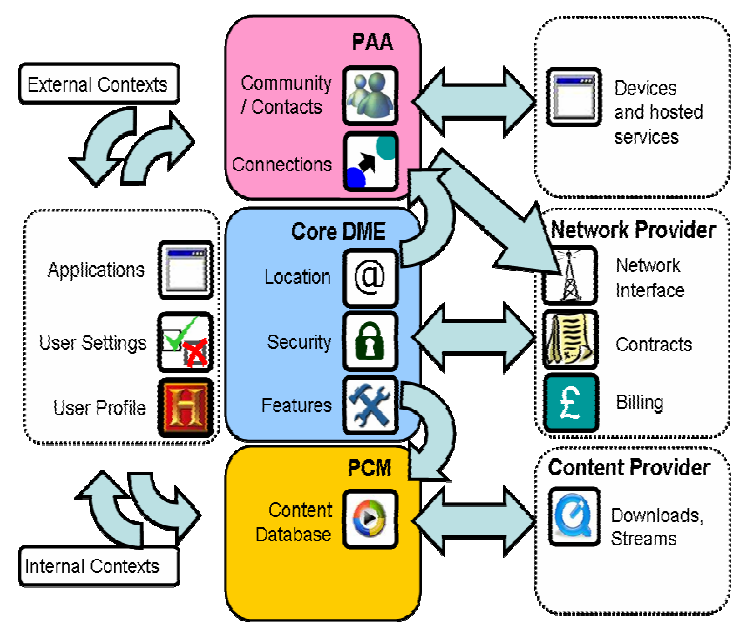

Figure 1. PAA within the PDE framework [1]

The PDE implements a user-centric view of multiple devices to access services in an inhomogeneous, dynamic networked environment. Devices can join and leave the PDE dynamically. It defines a Device Management Entity (DME) with a 2-tier hierarchy; a RootDME as the central point of contact and management and LocalDMEs for management of the sub-networks (home, car, corporate network etc). The DME handles topology management and ensures device connectivity.

The PAA monitors and manages the interfaces between the user and his environment. It aims at providing customized services tailored to the user's current context and seeks to free the user from repetitive decision making and device configuration issues. To perform this informed decision-making role, it collects and reasons upon a number of context variables, which include device, service, application and network context, apart from user context data. This requires definition of a device discovery and description module, which provides input to a context inference engine that seeks to come up with a comprehensive semantic contextual view of the user and the connected devices within the PDE.

\section{Device description in the PDE}

Previous work [8] on functional evaluation of existing industry-standard service discovery protocols, (Jini [9], UPnP [10], Salutation [11], SLP [12] and Bluetooth SDP [13]), has shown that UPnP is best suited to meet the challenges identified for current dynamic, mobile computing environments.

$\mathrm{UPnP}$ is a set of protocols designed to bring standards-based connectivity to ad-hoc networks, whether in the home, small business, public spaces or attached to the Internet [6]. Apart from its suitability to peer-to-peer network connectivity of smart appliances, wireless devices and PCs, it also offers rich description capabilities for advertising services through a declarative XML template, which is communicated through HTTP. XML offers a lightweight; crossplatform means to annotate device and service information. The device information provided is rudimentary with tags describing name, type, modelURL and URLs for service descriptions. It also includes a UUID (universally unique identifier) tag for uniquely identifying the device. Thus, the UPnP specification explicitly addresses the need for an identification mechanism for devices, which has been pointed out in [5]. The template also features a clear demarcation between the physical device and the hosted software services. Device description allows composition of devices, for instance, a mobile phone with screen, keypad etc; each capable of being discovered and used independently of the container device. Service descriptions define interactions with possible actions and corresponding inputs and outputs, which are related to state variables that represent the service's current status. State variables have defined types, scope for specifying default values as well as allowed values. Numerical values can be specified by valid ranges and allowed increments therein.

The proposed model contends that the allowedValue tag can be used for specifying formats associated with a modality service. Default values can be used to denote preferred choices. Additionally, the tight coupling between input and state variables means that the XML 
language can be used for verification of declarative content.

\subsection{Extending the UPnP standard}

Device hardware capabilities are necessary to form a complete description of a multimodal device and refine service descriptions. Pertinent to multimodal close to the UPnP template type in its simplicity, while being flexible enough to describe different kinds of modalities encountered. Figure 2 shows an XMLSpy IDE [14] representation of the extension.

< uuid > uniquely identifies a device. The <display> tag describes the screen in terms of its physical size and offered resolution. <userInput> is modeled on the MPEG-21 [15] standard for user interaction input

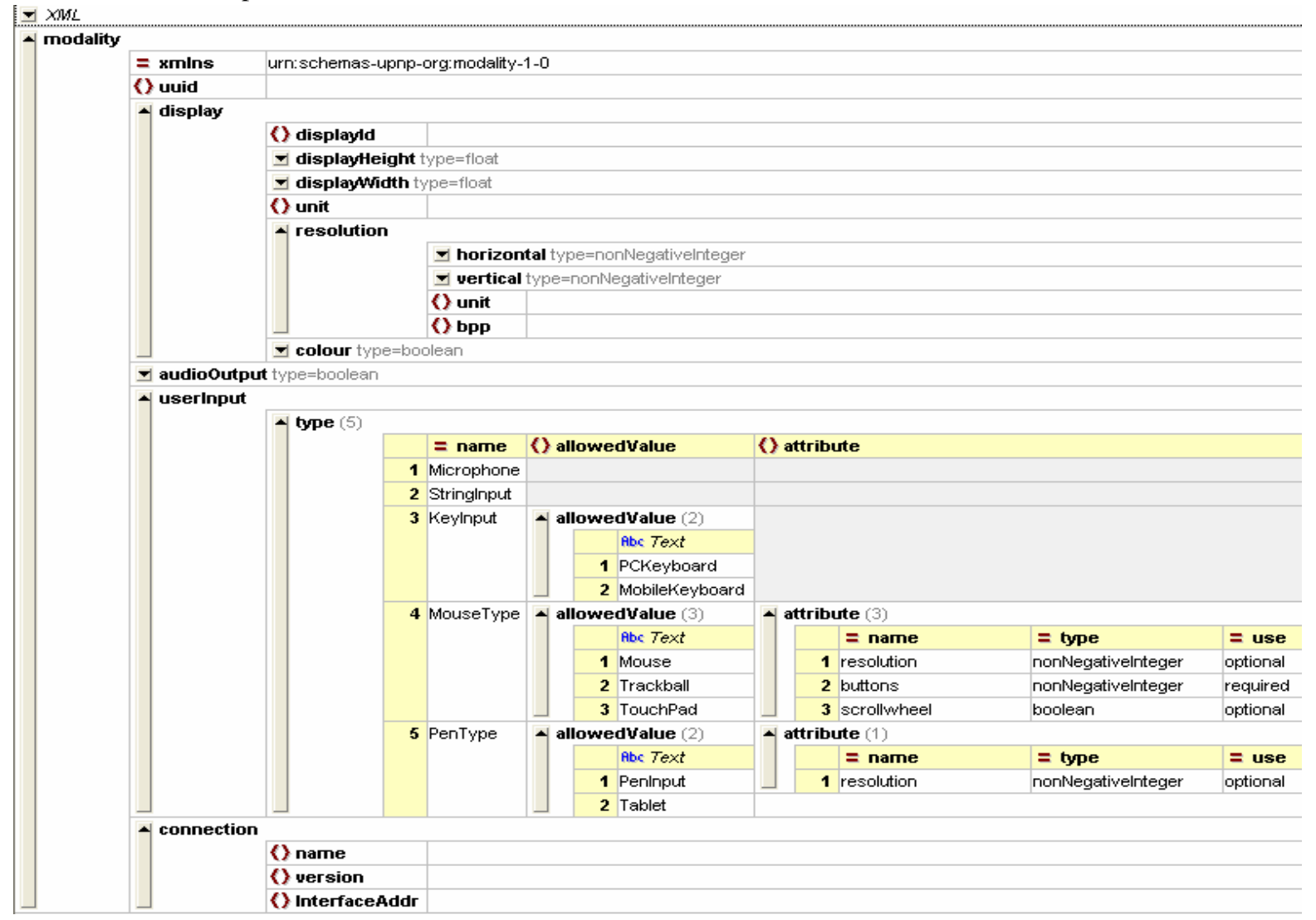

Figure 2. Modality extension to the UPnP template

interfaces, these fall into the following basic types: display capabilities that impact visual presentation (resolution, color capabilities, rendering formats), audio output presence and various types of user interaction input support. These feature types are those that can influence service presentation and personalization. Additionally, specification of device network interfaces can identify potential service delivery channels. The UPnP service template already supports facilities for specifying formats for display capabilities. However, it lacks provision for describing the other identified hardware capabilities. Based on this observation, an extension to the UPnP template is proposed that encapsulates various input and output modalities. The extension template is modeled to be syntax and captures user input modalities in generic types, each annotated with specific attributes. Finally, the network interfaces are captured and specified with name, version (to link to specific network interface capabilities) and the interface MAC address to uniquely identify the interface in the PDE.

\section{Implementation}

Implementation work has focused on developing comprehensive descriptions of discovered devices within the PDE. A complete description of a simulated electronic projection screen equipped with an Infrared (IR) port that communicates with an IR remote control is shown in Figures 3 and 4. 


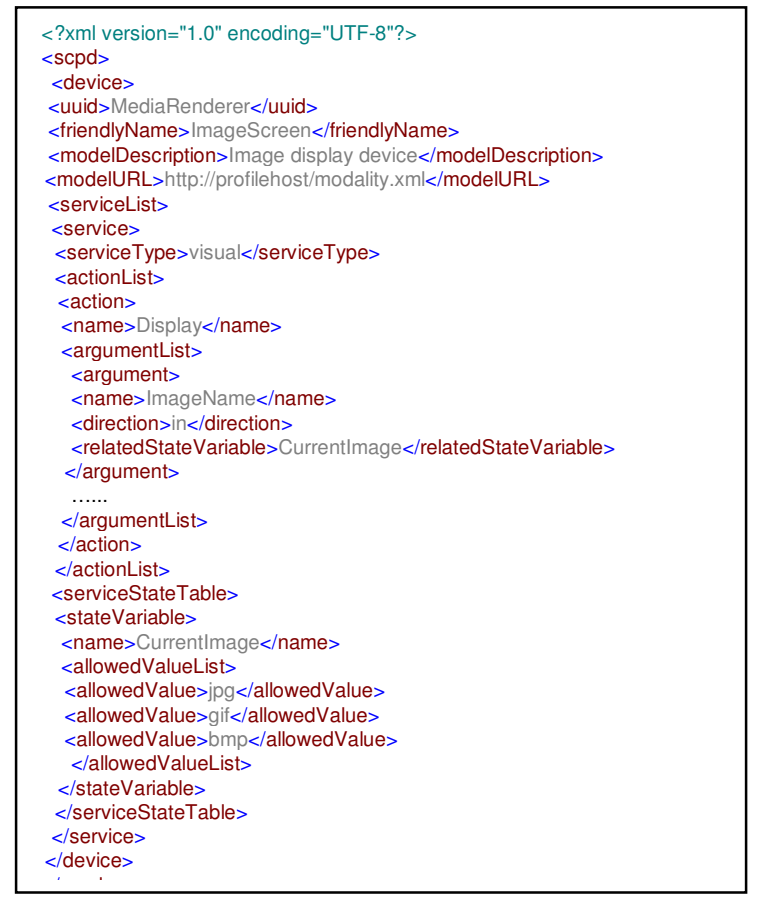

\section{Figure 3. Electronic screen service description}

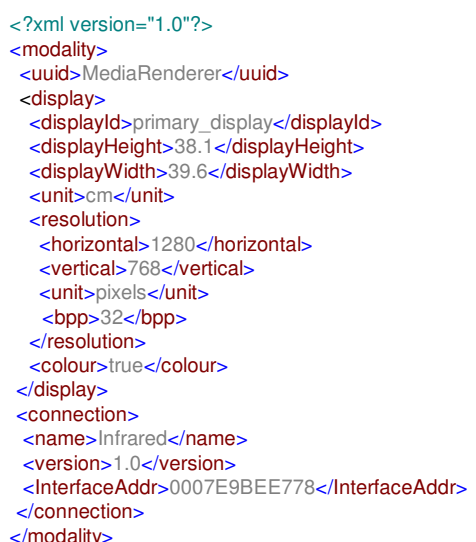

\section{Figure 4. Electronic screen hardware description}

The sequence of discovering devices and their descriptions consists of the following steps:

1. Devices within the PDE are discovered through the UPnP protocol.

2. The descriptions, which consist of a main file and separate XML description files for each hosted service, are retrieved through a HTTP GET command.

3. The descriptions are parsed and the relevant information extracted.
4. Relevant device information and descriptions of all hosted services are collated into a single XML file by first building a document object model (DOM) through Java XML APIs (as shown in Figure 3).

5. The URL of the hardware profile repository is extracted from the $<$ modelURL $>$ tag.

6. The modality description (Figure 4) is then retrieved through a Java RMI connection.

7. The combined description is then input into the framework for further processing.

\section{Discussion}

The model proposed in this paper is compared with two research community approaches, in terms of alignment with industry-standard discovery mechanisms and descriptive capabilities, respectively.

DReggie [16] is a service discovery architecture that builds upon Jini. Descriptions are modeled in DAML, using a common agreed ontology and services are required to register a DAML description as well as their Jini interface to a Jini Lookup Server. While this aids reasoning through the defined Prolog reasoner, requiring mobile devices to host ontology instances and the Jini requirement for devices to host the complete JVM calls into question its applicability to resource constrained devices. In contrast, the approach in this paper employs a lightweight XML template, which will be mapped to an ontology within the reasoning module.

The second approach, that comes close to that suggested in this paper, is Konark [17], a middleware for discovery and delivery of services in ad-hoc networks. It closely mirrors the UPnP template and introduces a keyword and properties tag to describe service characteristics. Service invocation and delivery is through SOAP calls, as it is with UPnP. Being targeted at m-commerce services, it doesn't include hardware descriptions.

Another related concept for service description is the OWL-S [18] ontology for describing Web services' properties and capabilities. However, it is more relevant to m-commerce services and not for multimodal services. The related OWL-S discovery protocol doesn't allow for extensions beyond its domain of business oriented services. Also, the OWL-S approach places no limitations on the discovery scope, whereas PDE applications have a strong focus on locality.

Taking into account the observations made above, the approach presented in this paper contends that inputs and outputs to services are implicitly handled in the UPnP template; formats and preferred values can 
be introduced by making use of allowed values on state variables. Furthermore, a layer of refinement is added to this by introducing the extension to UPnP with the modality profile template for describing generic devices. Use of the <modelURL $>$ tag of the UPnP template to specify the address of a repository that hosts the defined extensions follows the UAProf mechanism of devices publishing their capabilities to a web server. It is envisaged that device manufacturers will specify URLs of the device descriptions hosted on their websites in the appropriate format.

A prototype implementation [8] has shown that a user can be presented with a choice of compatible devices to display his content (still images, in this case) based on service annotation (based on image formats handled and supported transport protocols to transfer the image from a content store). Adding the hardware description will act as a further refinement to present the user with a more informed choice, for instance, indication as to which screen has a better resolution.

\section{Conclusions}

Since knowledge of available resources forms an integral part of a context-aware system, description of these resources is fundamentally important. This paper has proposed an extension to the UPnP descriptions with an aim to provide a complete description of multimodality interfaces. The implementation effort presented here and the scenario reported in [8] indicates that coupled with the UPnP discovery protocol, the proposed description model can provide a complete device discovery and description solution for a personal distributed environment.

Future work includes integration of the descriptions with ontology concepts to trigger contextual reasoning.

\section{Acknowledgement}

The work reported in this paper has formed part of the Ubiquitous Services Core Research Programme of the Virtual Centre of Excellence in Mobile \& Personal Communications, Mobile VCE, www.mobilevce.com. This research has been funded by the DTI-led Technology Programme and by the Industrial Companies who are Members of Mobile VCE. Fully detailed technical reports on this research are available to Industrial Members of Mobile VCE.

\section{References}

[1]J. Bush, J. Irvine, and J. Dunlop, "Personal Assistant Agent and Content Manager for Ubiquitous Services," presented at International Symposium on Wireless Communication Systems (ISWCS), Valencia, Spain, 2006.

[2]W3C, "Composite Capability/Preference Profiles (CC/PP): Structure and Vocabularies," 2002. [Online].

Available: http://www.w3.org/TR/2004/REC-CCPPstructvocab-20040115

[3]Open Mobile Alliance (OMA), "User Agent Profile," May 20, 2003. [Online]. Available:

http://www.openmobilealliance.org

[4]R. Eisinger, M. G. Manzato, and R. Goularte, "Devices Description for Context-Based Content Adaptation," in Third Latin American Web Congress (LA-WEB'05). Buenos Aires, Argentina: IEEE, 2005.

[5]R. Kernchen, M. Boussard, R. Haensel, and K. Moessner, "Device description for mobile multimodal interfaces," in 15th IST Mobile \& Wireless Communication Summit. Mykonos Island, Greece, 2006.

[6]H. Li and H. Wang, "A Method of Service Description and Discovery in Pervasive Computing Environments," presented at 1st International Symposium on Pervasive Computing and Applications, Xinjiang, China, 2006.

[7]O. K. Zein and Y. Kermarrec, "An approach for service description and a flexible way to discover services in distributed systems," presented at International Conference on Information Technology: Coding and Computing (ITCC), 2005.

[8]S. De and K. Moessner, "Context gathering in Ubiquitous Environments: Enhanced Service Discovery," presented at Third Workshop on Context Awareness for Proactive Systems (CAPS'07), Guildford, UK, 2007.

[9]Sun Microsystems, "Jini Technology Core Platform Specification," v. 2.0 ed, 2003. [Online]. Available:

www.sun.com/software/jini/specs/core2_0.pdf.

[10] UPnP Forum, "UPnPTM Device Architecture," December 2003. [Online]. Available:

http://www.upnp.org/resources/documents/CleanUPnPDA10 1-20031202s.pdf.

[11] Salutation Consortium, "Salutation Architecture Specification," 1999.

[12]E. Guttman et al., "Service Location Protocol," IETF RFC 2608, v. 2, June 1999.

[13]Bluetooth SIG, "Specification of the Bluetooth System," 2003.

[14]XMLSpy. [Online]. Available:

http://www.altova.com/products/xmlspy/xml_editor.html

[15]BS ISO/IEC, "Information Technology — Multimedia Framework (MPEG-21) — Part 7: Digital Item Adaptation," vol. 21000-7:2004, 2004.

[16]D. Chakraborty, F. Perich, S. Avancha, and A. Joshi, "Dreggie: Semantic Service Discovery for M-commerce Applications," presented at Workshop on Reliable and Secure Applications in Mobile Environment, 20th Symposiom on Reliable Distributed Systems, 2001.

[17]L. Choonhwa, A. Helal, N. Desai, V. Verma, and B. Arslan, "Konark: A system and protocols for device independent, peer-to-peer discovery and delivery of mobile services," IEEE Transactions on Systems, Man and Cybernetics, Part A, vol. 33, pp. 682-696, 2003.

[18] W3C, "OWL-S: Semantic Markup for Web Services," W3C Member Submission, 22 November 2004. 